Apidologie, 1977, 8 (4), 295-304.

\title{
BAU UND FEINBAU DER NEKTARIEN UND DER MECHANISMUS DER NEKTARSEKRETION
}

\author{
Structure et ultrastructure des nectaires \\ et mécanisme de la sécrétion nectarifère
}

Eberhard SCHNEPF (1)

Lehrstuhl für Zellenlehre, Universität Heidelberg

\section{SUMMARY}

STRUCTURE AND FINE STRUCTURE OF NECTARIES

AND THE MEGHANISM OF NEGTAR SEGRETION

Based on ultrastructural studies of different nectaries different authors suggested different routes for the prenectar to move into the nectary and finally to the surface of the plant. The ultrastructural observations seem to confirm the various suggestions. Cytological and physiological studies of Bromeliacean nectaries indicate that the bulk of the nectar here is secreted by the Golgi-apparatus but that simultaneously some components may take the apoplasnic route.

\section{ZUSAMMENFASSUNG}

Auf Grund von Ultrastrukturuntersuchungen an verschiedenen Nektarien kamen verschiedene Autoren zu verschiedenen Vorstellungen über den Weg des Pronektars in das Nektarium und schließich an die Oberfäche der Pflanze. Die feinstrukturellen Beobachtungen scheinen die verschiedenen vorgeschlagenen Wege zu bestätigen. Nach cytologischen und physiologischen Befunden an den Nektarien von Bromeliaceen ist es wahrscheinlich, daß hier der Nektar hauptsächlich durch den Golpi-Apparat sezerniert wird, daß aber einige Nektarkomponenten gleichzeitig im Apoplasten wandern.

(1) Mit Unterstützung der Deutschen Forschungsgemeinschaft. 


\section{I. - Einleitung}

Nektarien kommen an den verschiedensten oberirdischen Pflanzenteilen und in den verschiedensten Formen vor (Übersichten z.B. bei J. G. Zimmermann 1932, Feldhofen 1933 und Sperlich 1939), auch bei Farnen (Lüttge 1961). Es ist daher unmöglich, hier eine umfassende Übersicht über den Bau und Feinbau der Nektarien zu geben. Der großen Mannigfaltigkeit im makroskopisch-morphologischen Bereich steht eine gewisse Einheitlichkeit auf dem zellulären und subzellulären Niveau gegenüber. Alle Zellen unterscheiden sich in ihren Strukturen eigentlich nur quantitativ, aber kaum qualitativ. Unser Problem ist es, zu versuchen, trotz dieser Uniformität aus der Analyse der Nektarienstruktur auf den Mechanismus der Nektarsekretion zu schließen (vergl. Schnepf 1969, Lüttge 1971, Lüttge und Schnepf 1976). Diese Aufgabe ist aus mehreren methodischen Gründen nicht leicht.

Deshalb gibt es heute noch kein klares, allgemein gültiges Konzept über den Mechanismus der Nektarsekretion. Ich möchte weiter gehen und zeigen, daß es solch ein allgemein gültiges Konzept gar nicht geben kann (vergl. LÜTTGE und SCHNEPF 1976).

\section{II. - BAU DER NEKTARIEN}

Das zeigt schon ein Blick auf den Bau der Nektarien. Nektarien sind häufig makroskopisch gut zu erkennen (gestaltete Nektarien). Sie bestehen dann meistens aus einem Drüsenepithel und einem Nektarienparenchym; in einigen Fällen ist anstelle eines Drüsenepithels ein Rasen aus Drüsenhaaren ausgebildet. Der Nektar tritt durch die Epithelzellen oder die Köpfchenzelle des Drüsenhaares nach außen. Dabei ist manchmal — wenn es sich um Drüsenhaare handelt, in der Regel - durch Cutinisierungen von Zellwandabschnitten dafür gesorgt, daß der Pronektar auf dem Weg in die sekretorische Zelle den Protoplasten einer Zelle (Scheidenzelle, vergl. die Endodermis) passieren muß.

Das ist aber durchaus nicht bei allen Nektarien so und trifft vor allem nicht bei den « ungestalteten Nektarien » zu, die makroskopisch nicht differenziert sind, zum Teil zwar ein Nektarienparenchym haben, in manchen Fällen (z.B. bei den Kelchblatt-Nektarien von Paeonia) aber nicht einmal das. Der Nektar tritt bei diesen Nektarien durch modifizierte Spaltöffnungen nach außen (J. G. Zimmermann 1932, Frey-Wyssling und Häusermann 1960).

Allen Nektarien ist gemeinsam, daß in ihrer Nähe Leitbündel verlaufen. Der Nektar stammt im Wesentlichen aus dem Phloem, wird aber bei seiner Sekretion noch modifiziert (Agthe 1951, Ziegler 1965, de Fekete et al. 1967). 
Um die Nektarsekretion zu verstehen, - und damit auch den Bau und Feinbau der Nektarien -, müssen wir untersuchen, auf welchen Wegen der Pronektar von der Siebröhre in das Nektarium wandert, um dann als Nektar auszutreten.

\section{III. - MÖGLICHE WEGE DER NEKTARSEKRETION}

In Abb. $I$ ist in sehr vereinfachter Form dargestellt, welche Wege grundsätzlich denkbar sind. Grau dargestellt ist das Cytoplasma, weiß der « free space ) in den Zellwänden (Apoplast) sowie die nicht-plasmatische Phase (im ER und im Golgi-Apparat; die Vacuolen sind weggelassen, sie würden ebenfalls weiß markiert sein müssen). Alle Zellen sind durch Plasmodesmen miteinander verbunden, durch die das Cytoplasma, aber auch das ER der benachbarten Zellen miteinander kommunizieren. Der Ausdruck “ Symplast 》 für dieses System simplifiziert eigentlich die Verhältnisse in unzulässiger Weise, weil er sowohl die plasmatische wie die nichtplasmatische intrazelluläre Phase einschließt. Die Zucker werden im Cytoplasma der Siebröhren herantransportiert. Um nach außen zu kommen, müssen sie wenigstens einmal eine cytoplasmatische Membran passieren. Diese Membranpassage ist im Fall der Zucker vermutlich ein aktiver Transportprozess und kann dann gegen ein

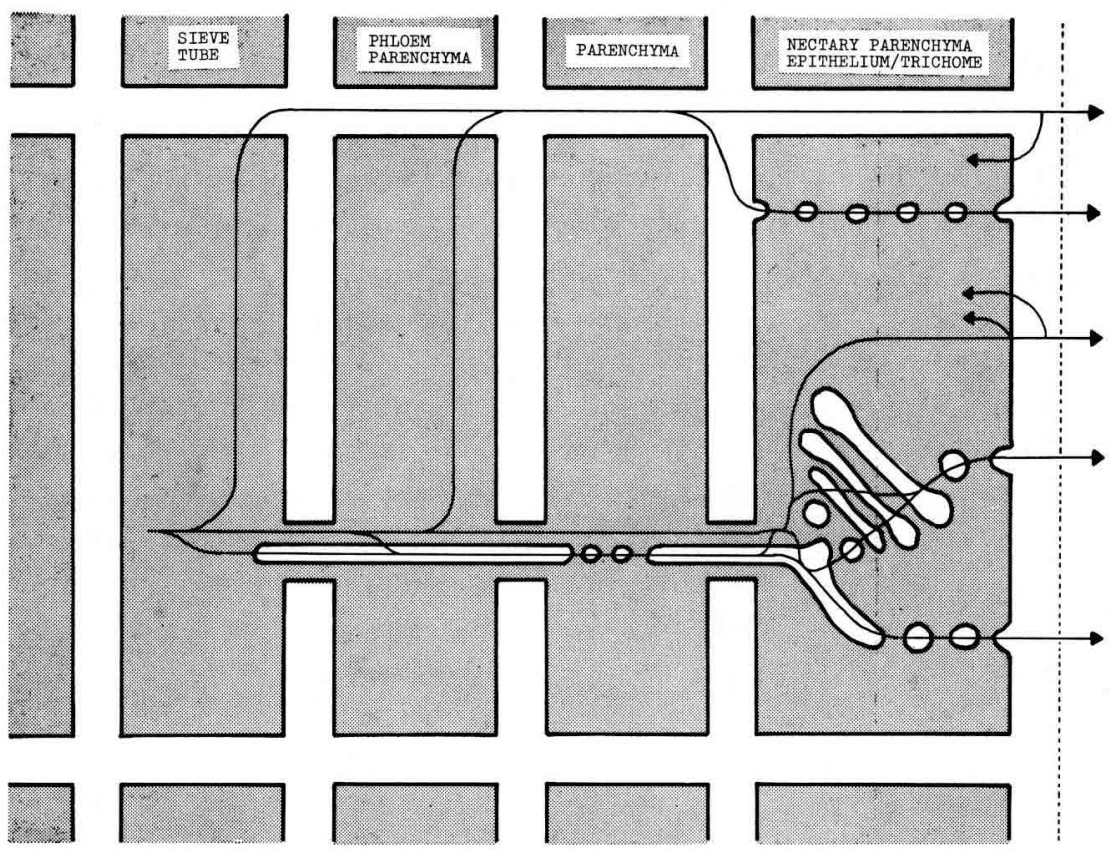

Aвв. 1. - Mögliche Wege des Pronektars bei der Sekretion, schematisch.

Fic. 1. - Schêma des chemins possibles du pronectar lors de la sécrétion. 
Konzentrationsgefälle ablaufen (s. das folgende Referat von LüTtge). Es ist allerdings nicht ganz auszuschließen, obwohl wenig wahrscheinlich, daß Membranen oder vielleicht nur bestimmte Membranbezirke für Zucker permeabel sind, daß es c Zuckerlecks » gibt. Das Wasser, mengenmäßig die Hauptkomponente des Nektars, kann vermutlich die Membranen immer leicht permeieren.

Als Ort für die Membranpassage der Zucker kommt das Plasmalemma der Sicbröhre selbst oder, wenn der Nektar durch den Plasma-Kanal der Plasmodesmen in Geleitzellen, Phloemparenchymzellen usw. gewandert ist, das Plasmalemma dieser Zellen in Frage. Der Pronektar würde dann weiter im Apoplasten wandern, wobei er durch Resorptionen (LüTTGE 1962) oder enzymatische Aktivitäten der Zellen (M. ZimmermanN 1954), an denen er vorbeifließt, verändert werden kann. Außerdem könnten die Drüsenepithelzellen die Substanzen aus dem Apoplasten aufnehmen und via Cytopempsis oder nach zweifacher Membranpassage nach außen befördern. Möglich, aber nicht sehr wahrscheinlich (vergl. Gunving und Hughes 1976) ist eine wiederholte Passage der Plasmalemmen.

Zwei grundsätzlich andere Wege bieten die Plasmodesmen. Das cytoplasmatische Kontinuum reicht von der Siebröhre bis zur Epithelzelle. Die Membranpassage kann also in das Plasmalemma der Epithelzelle verlegt werden (eccrine Sekretion). Durch selektiven Transport zusammen mit Zukkerumwandlungen und/oder Rückresorptionen kann dabei die Nektarzusammensetzung verändert werden. Es ist aber auch möglich, daß in den Drüsenzellen die Kompartimente des ER oder des Golgi-Apparates die Zucker aufnehmen und sie dann exocytotisch exportieren. Die Membranpassage ist dann in das Zellinnere verlegt (granulocrine Sekretion).

Durch den Desmotubulus der Plasmodesmen steht aber auch das ER benachbarter Zellen in Verbindung. Wenn das Siebröhren-ER beladen wird, kann der Pronektar kontinuierlich oder diskontinuierlich (über ER-Vesikel) ohne weitere Membranüberquerung bis in die Epithelzelle und darüber hinaus bis nach außen transportiert werden, direkt, d.h. über Fusionen von ERElementen mit dem Plasmalemma, oder indirekt, d.h. nach einer Passage des Golgi-Apparates über Golgi-Vesikel. Es ist aber auch vorstellbar, daß die Zucker das ER in den Epithelzellen verlassen und dann eccrin durch das Plasmalemma treten. Auf allen diesen Wegen kann das Prosekret leicht verändert werden.

\section{IV. - FEINSTRUKTUR VON NEKTARIEN UND SEKRETIONSMECHANISMEN}

Bei der elektronenmikroskopischen Untersuchung verschiedener Nektarien kamen die Autoren, wie nach der Anatomie der Nektarien zu erwarten war, zu ganz verschiedenen Vorstellungen über den Sekretionsprozeß, die im 
folgenden, z.T. etwas schematisiert, dargestellt werden; dabei haben sich meistens die Autoren selbst viel vorsichtiger ausgedrückt, als es hier gedrängt dargestellt werden kann.

Vasiliev $(1969,1971,1972)$ untersuchte u.a. die Nektarien von Acer, Ranunculus, Heracleum, Convolvolus und Cucumis. Er beobachtete keinerlei Sekretvesikel, weder vom ER noch vom Golgi-Apparat. Nur in einigen Fällen schien der Golgi-Apparat aktiv zu sein, aber offenbar nicht in Zusammenhang mit der Nektarausscheidung. Im Gegenteil : in den meisten aktiven Nektarien war der Golgi-Apparat nur schlecht entwickelt. In einigen dieser Nektarien war das ER recht ausgedehnt; VASILIEv meint aber, daß das mit einer Beteiligung dieses Organells an der Produktion von Hormonen zusammenhängt. Es gab außerdem keine Zeichen für eine eccrine Sekretion (d.h. keine Vergrößerung des Plasmalemmas durch Wandprotuberanzen, s.u.). Plasmodesmen waren besonders in der aktiven Phase selten.

Daraus schließt Vasiliev, daß die Zucker aus den Siebröhren in die PhloemParenchymzellen wandern und von diesen in den Apoplast sezerniert werden. Der Pronektar fließt dann in den Zellwänden nach außen und wird dabei durch Reabsorptionen und durch Enzyme wie Invertase verändert. Das Nektarium spielt also beim eigentlichen Sekretionsprozeß keine Rolle.

Ganz im Gegensatz dazu ist bei den Trichom-Nektarien von Abutilon der Weg im Apoplast zu den Drüsenköpfchen durch Cutinisierungen in der Wand der Stielzelle versperrt. Der Pronektar müßte also mehrfach die Plasmalemmen durchqueren, was aber nach GunNing und Hughes (1976) aus energetischen Gründen (vergl. Findlay et al. 1971) nicht wahrscheinlich ist, oder er kommt über die zahlreichen Plasmodesmen in die Köpfchenzellen. Da die Drüsenzellen ein sehr gut entwickeltes ER enthalten, das sich zudem in Zusammenhang mit der Sekretionsintensität verändert, neigen Mercer und RATHgeber (1962, s.a. Findlay und Mercer 1971a und b, Findlay et al. 1971, Reed et al. 1971) dazu, ihm eine Rolle beim Prosekrettı ansport zuzuschreiben. Sie konnten aber keine sicheren Hinweise auf eine Exocytose via ER-Vesikel finden, obwohl auch dieser Prozeß nicht auszuschließen ist. Die Dictyosomen erscheinen inaktiv. Die Autoren vermuten daher eher, daß das Plasmalemma der Köpfehenzellen direkt von den Zuckern passiert wird, entweder durch einen aktiven Transport oder passiv, wobei dann die Sekretion durch das aktive Beladen der Drüsenzelle getrieben würde. Die Spaltung der ausgeschiedenen Saccharose zu Glucose und Fructose könnte einen osmotischen Gradienten erzeugen, der den Efflux von Wasser und Zucker verstärkt. Das Sekret tritt schließlich durch Poren in der Cuticula aus.

Untersuchungen und Überlegungen von GunNING und Hughes (1976) an diesen Nektarien zeigen, daß die Dimension und Zahl der Plasmodesmen im Drüsenstiel ausreichen, um den Fluß von Zuckern und Wasser zu leiten. 
Dabei hat der Plasmaring in den Plasmodesmen eine größere Querschnitts fläche als der Desmotubulus. Das bei einem Fluß einer Zuckerlösung in dem einem der beiden Kompartimente zu erwartende Mitströmen von kleinen Molekülen und Ionen könnte durch einen Gegentransport, eventuell im anderen Kompartiment, wieder kompensiert werden. Allerdings ist ein solcher Ko-Transport wohl nur zu erwarten, wenn der Fluß im Plasmaring stattfindet, denn der Inhalt des ER ist sicher leicht regulierbar. Der Reichtum der Drüsenzellen, aber nicht nur der Köpfchenzellen, an Mitochondrien deutet darauf hin, daß hier aktive Zuckertransportprozesse ablaufen (REED et al. 1971, GunNing und Hughes 1976).

Ähnliche Beobachtungen machten FaHN und Rachmilevitz (1970) und Rachmilevitz und Fahn (1973, 1975) an den Nektarien von Lonicera, Vinca, Citrus und Tropaeolum. Eine Sperre im Apoplasten gibt es hier z. T. nicht. Dennoch verbinden sehr viele Plasmodesmen das Phloem mit den Drüsenzellen. Die aktiven Nektarien enthalten ein sehr ausgedehntes rauhes und glattes ER, die ineinander übergehen. Die ER-Zisternen sind oft aufgebläht, was andeutet, daß sie viel osmotisch wirksames Material enthalten, vermutlich Zucker, worauf auch die autoradiographischen Befunde von FAHN und RACHMILEvitz (1975) hinweisen.

Vom glatten ER werden Vesikel gebildet; diese scheinen mit dem Plasmalemma zu fusionieren. Eine relativ große Zahl von multivesikulären Körpern könnte ebenfalls mit der Exocytose in indirektem Zusammenhang stehen : sie zeigen vielleicht den Rücktransport von Membranmaterial an, der als Kompensation für den Einbau der Vesikelmembranen in das Plasmalemma notwendig ist. Bei Lonicera und Tropaeolum war auch der Golgi-Apparat aktiv, doch steht das vermutlich bei Lonicera nicht mit der Nektarsekretion, sondern mit der Bildung von Wandprotuberanzen in Zusammenhang.

Solche Wandprotuberanzen sind in vielen Septalnektarien von Liliifloren (SchnepF 1964), aber z.B. auch in den Trichom-Nektarien von Vicia faba (Wrischer 1962) zu finden. Bei Gasteria und Aloe werden sie kurz vor Beginn der Nektarsekretion gebildet und bald danach wieder abgebaut (SchnepF und Pross 1976). Die vielen Plasmodesmen zwischen Drüsenepithelzellen und Phloem sind ein Hinweis dafür, daß der Pronektar im Symplast wandert. Die Vergrößerung des Plasmalemmas durch die Wandprotuberanzen wurde als Indiz dafür genommen, daß die Zucker eccrin, d.h. direkt durch das Plasmalemma, sezerniert werden (Schnepf 1964, 1969, vergl. Zandonella 1972). Solche direkten Membranpassagen sind für alle Transferzellen charakteristisch (Gunning und Pate 1974). Allerdings haben die Drüsenepithelzellen ein gut entwickeltes ER und viele Dictyosomen, und Heinrich (1975a) fand autoradiographisch Zucker im ER und anderen inneren nichtplasmatischen Kompartimenten. Es ist also sehr wohl möglich, daß die Zellen auch exocytotisch 
aktiv sind, was Heinrich (1975b) auch auf Grund von cytochemischen Untersuchungen über die Lokalisation von Phosphatasen postuliert. Vielleicht steht die Radioaktivität in diesen Kompartimenten aber auch nur mit dem Zuckerimport oder einer Zuckerspeicherung, und nicht mit dem Zuckerexport in Zusammenhang.

Bei den Septalnektarien von Bromeliaceen, bei denen die Drüsenepithelzellen keine Wandprotuberanzen haben, gibt es aber Befunde, die sehr dafür sprechen, daß der Golgi-Apparat an der Nektarsekretion beteiligt ist (Benner und Schnepf 1975, Benner 1976, vergl. Eymé 1967, Rachmilevitz und FAHN 1975). Die Drüsenepithelzellen enthalten viele Dictyosomen. In der aktiven Phase, und nur dann, sind sie hypersekretorisch und bilden viele große sekretorische Golgi-Vesikel. Wenn man die Nektarsekretion durch Verdunkelung oder Abkühlung hemmt, findet man nur inaktive Dictyosomen. Ebenso inhibieren Kaliumcyanid $\left(10^{-3} \mathrm{M}\right)$, Dinitrophenol $\left(10^{-3} \mathrm{M}\right)$, Cytochalasin B $(1 \mu \mathrm{g} / \mathrm{ml})$, Tetraäthylblei $\left(10^{-4} \mathrm{M}\right)$, Desoxyglucose $(5 \%)$, aber auch einige Farbstoffe wie Natrium-Fluorescein $(0,1 \%)$ und Acridinorange $(0,1 \%)$ sowohl die Nektarsekretion wie die Aktivität der Dictyosomen. Diese Übereinstimmung kann nur dadurch erklärt werden, daß der Golgi-Apparat Nektar sezerniert.

Wenn man voraussetzt, daß der Nektar ausschließlich durch GolgiVesikel ausgeschieden wird, läßt sich deren “ Lebensdauer ” aus der pro Minute abgegebenen Sekretmenge und dem Volumen der Golgi-Vesikel in den Drüsenzellen abschätzen. Der Wert, 2-3 Minuten in der aktivsten Phase, stimmt sehr gut mit den Ergebnissen überein, die an Schleimdrüsen (SchNepF 1961) und bei anderen Objekten (z.B. Brown 1969) gewonnen wurden.

Es ist allerdings die Frage, ob die Voraussetzung richtig ist, daß der Nektar nur durch Golgi-Vesikel ausgeschieden wird. Appliziert man abgeschnittenen Blüten über den Blütenstiel verschiedene Substanzen und prüft, ob sie im Nektar erscheinen, so findet man, daß Farbstoffe und Aminosäuren nicht austreten. Wohl aber tauchen Fremdzucker wie Galaktose, Melibiose, Maltose, Raffinose und Ribose im Sekret auf, sogar Desoxyglucose, die allerdings, wie erwähnt, die Sekretion im Gegensatz zu den anderen Zuckern stark hemmt. Dabei dauert es etwa 90 Minuten, bis die Fremdzucker im Nektar nachweisbar sind.

Für diese Befunde gibt es zwei mögliche Erklärungen. Wenn man davon ausgeht, daß der Pronektar allein im Symplasten vom Phloem zu den Drüsenzellen transportiert wird, müssen auch die Fremdzucker die Membran (oder die Membranen) passieren können. Das ist bei der Annahme einer spezifischen Zuckerpumpe jedoch schwer zu verstehen. Und daß spezifische Zuckerpumpen aktiv sind, zeigen die Wirkungen von Desoxyglucose; dieser Zucker hemmt kompetitiv die Glucose-« Pumpe ». 
Wenn man allerdings die Zahl der Plasmodesmen zur Sekretionsintensität in Beziehung setzt, erkennt man, daß sie ausreichen würden, um den Symplasma-Transport zu bewältigen. Aus den Angaben von Gunning und Hughes (1976) läßt sich errechnen, daß bei den Trichomnektarien von Abutilon $0,02 \mu^{3}$ Pronektar pro Sekunde durch einen Plasmodesmos fließt. In den BillbergiaNektarien sind es nur $0,003 \mu \mathrm{m}^{3}$.

Es ist aber auch möglich, daß einige Nektarkomponenten, hier die Fremdzucker, im Apoplast wandern. Sie könnten in diesen Fall aus dem Xylem stammen und einen osmotisch, durch den ausgeschiedenen Nektar getriebenen Wasserstrom anzeigen. Zucker, die dabei mitflössen, würden die Wände der Parenchym- und Epithelzellen passieren, Aminosäuren resorbiert werden. Kalkulationen, die im Apoplasten zur Verfügung stehende Durchströmungsfläche abzuschätzen, führten zu dem von uns nicht erwarteten Ergebnis, daß sie nur ungefähr dreimal so groß ist wie die im cytoplasmatischen Kompartiment der Plasmodesmen.

\section{v. - SCHLuss}

Es ist also nicht auszuschließen, daß sogar in ein und demselben Nektarium verschiedene Wege der Nektarsekretion nebeneinander vorkommen. Das Problem der Zuckerausscheidung durch Nektarien kann also von der Pflanze auf verschiedene Weise gelöst werden. Dennoch ist klar, daß die Sekretion auf wenige Grundprozesse zurückzuführen ist; vor allem ist dabei die Membranpassage wichtig.

Eingegangen im April 1977. Reçu pour publication en avril 1977.

\section{RÉSUMẼ}

En se basant sur des études de l'ultrastructure de différents nectaires, divers auteurs ont suggéré l'existence pour le pronectar de plusieurs chemins vers le nectaire et finalement vers la surface de la plante. Les observations faites sur l'ultrastructure semblent confirmer les diverses hypothèses. Des études cytologiques et physiologiques des nectaires de Broméliacées indiquent que la majeure partie du nectar est dans ce cas sécrétée par l'appareil de Golgi mais qu'en même temps certains composants peuvent emprunter la voie des apoplastes.

\section{LITERATUR}

Agthe C., 1951. - Über die physiologische Herkunft des Pflanzennektars. Ber. Schweiz. Bot. Ges., 61, 243-273.

Benner U., 1976. - Zur Morphologie und Physiologie der Nektarsekretion bei Bromeliaceen. Diss. Univ. Heidelberg. 
Benner U. u. Schnepf E., 1975. - Die Morphologie der Nektarausscheidung bei Bromeliaceen : Beteiligung des Golgi-Apparates. Protoplasma, 85, 337-349.

Brown R. M., 1969. - Observations on the relationship of the Golgi apparatus to wall formation in the marine chrysophycean alga, Pleurochrysis scherffelii Pringsheim. J. Cell Biol., 41, 109-123.

Eуме́ J., 1967. - Nouvelles observations sur l'infrastructure de tissus nectarigènes floraux. Botaniste, 50, 169-183.

Fahn A. u. Rachmilevitz T., 1970. - Ultrastructure and nectar secretion in Lonicera japonica. Bot. J. Linnean Soc., 63, Suppl. 1 : New Research in Plant Anatomy, 51-56.

FahN A. u. Rachmilevitz, T., 1975. - An autoradiographical study of nectar secretion in Lonicera japonica Thunb. Ann. Bot., 39, 975-976 (1975).

Fekete M. A. R. De, Ziegler H. u. Welf R., 1967. - Enzyme des Kohlenhydratstoffwechsels in Nektarien. Planta, 75, 125-138.

Feldhofen E., 1933. - Beiträge zur physiologischen Anatomie der nuptialen Nektarien aus den Reihen der Dikotylen. Beih. Bot. Zbl., i Abt., 50, 459-634.

Findeay N. u. Mercer F. V., 1971a, - Nectar production in Abutilon. I. Movement of nectar through the cuticle. Austr. J. Bicl. Sci., 24, 647-656.

Findlay N. u. Mercer, F. V., 1971 b. - Nectar production in Abutilon. II. Submicroscopic structure of the nectary. Austr. J. Biol. Sci., 24, 657-664.

Findlay N., Reed M. L. u. Mercer, F. V., 1971. - Nectar production in Abutilon. III. Sugar secretion. Austr. J. Biol. Sci., 24, 665-675.

Frey-Wysshing A. u. Häusermann E., 1960. — Deutung der gestaltlosen Nektarien. Ber. Schweiz. Bot. Ges., 70, 150-162.

Gunning B. E. S. u. Hughes J. E., 1976. - Quantitative assessment of symplastic transport of pre-nectar into the trichomes of Abutilon nectaries. Austr. J. Plant Physiol., 3, 619-637.

Herneich G., 1975a. — Über den Glucose-Metabolismus in Nektarien zweier Aloe-Arten und über den Mechanismus der Pronektar-Sekretion. Protoplasma, 85, 351-371.

Heinnich G. 1975b. - Über die Lokalisation verschiedener Phosphatasen im Nektarium von Aloe. Cytobiologie 11, 247-263.

LüTTge U., 1961. - Ủber die Zusammensetzung des Nektars und den Mechanismus seiner Sekretion. I. Planta, 56, 189-212.

LüTtge U., 1962. — Über die Zusammensetzung des Nektars und den Mechanismus seiner Sekretion. III. Mitteilung. Die Rolle der Rückresorption und der spezifischen Zuckersekretion. Planta, 59, 175-194.

Lütrge U. 1971. - Structure and function of plant glands. Annu. Rev. Plant Physiol., 22, 23-44.

Lüttge U. u. SchnepF E., 1976. - Elimination processes by glands. Organic substances. In Lüttge U. u. Pitman U. G. : Encyclopedia of Plant Physiology, New Series, Vol. 2. Transport in Plants II. Part B. Tissues and organs. Berlin, Springer, 244-277.

Mercer F. V. u. Rathgeber N., 1962. - Nectar secretion and cell membranes. 6. Int. Congress Electron Microscopy, Philadelphia, Vol. 2, WW 11.

Rachmilevitz T, u. FAHN A., 1975. - The floral nectary of Tropaeolum majus L. -- The nature of the secretory cells and the manner of nectar secretion. Ann. Bot., 39, 721-728.

Reed, M. L., Findlay N. u. Mercer F. v, 1971. - Nectar production in Abutilon. IV. Water and solute relation. Austr. J. Biol. Sci., 24, 677-688.

SCHNEPF E., 1961. - Quantitative Zusammenhänge zwischen der Sekretion des Fangschleimes und den Golgi-Strukturen bei Drosophyllum lusitanicum. Z. Naturforsch., 16 b, 605-610.

Schnepf E., 1964. — Zur Cytologie und Physiologie pflanzlicher Drüsen. 4. Teil. Licht und elektronenmikroskopische Untersuchungen an Septalnektarien. Protoplasma, 58, 137-171.

Schnepf E., 1969. - Sekretion und Exkretion bei Pflanzen. Protoplasmatologia, VIII, 8. Wien, Springer.

Schnepf E. u. PRoss E., 1976. - Differentiation and redifferentiation of a transfer cell : development of septal nectaries of Aloe and Gasteria. Protoplasma, 89, 105-115.

Sperlich A., 1939. - Das tropische Parenchym. Exkretionsgewebe. In. LINsBauer K. Handbuch der Pflanzenanatomie 1. Abt. 2. Teil Bd. IV B. Borntraeger, Berlin. 
VASILIJEV A. E., 1969. - Submicroscopic morphology of nectary cells and problems of nectar secretion (russisch). Akad. Nauk SSSR, Bot. Z., 54, 1015-1031.

VAsiLIJEV A. E., 1971. - New data on the ultrastructure of the cells of the flower nectary (russisch). Akad. Nauk SSSR, Bot. Z., 56, 1292-1306.

VASILIJEv E. E., 1972. - The ultrastructure of the neetary cells of Cucumber (russisch). Akad. Nauk SSSR, Citologija, 14, 405-415.

Wrischer M., 1962. - Elektronenmikroskopische Beobachtungen an extrafloralen Nektarien von Vicia faba L. Acta Botan. Croat., 20/21, 75-94.

ZANDONELLA P., 1972. - Le nectaire floral des Centrospermales. Localisation, morphologie, anatomie, histologie, cytologie. These, Univ. Lyon.

Zregler H., 1965. - Die Physiologie pflanzlicher Drüsen. Ber. Dtsch. Bot. Ges., 78, 466-477.

Zimmermann J. G., 1932. -- Über die extrafloralen Nektarien der Angiospermen. Beih. bot Zbl., 1 Abt., 49, 99-196.

ZimmermanN M., 1954. - Über die Sekretion saccharosespaltender Transglucosidasen im pflanzlichen Nektar. Experientia, 10, 145-146. 